\title{
Natural orifices transluminal endoscopic surgery (NOTES) and other allied "ultra" minimally invasive procedures: are we loosing the plot?
}

\author{
Luigi Boni · Gianlorenzo Dionigi · Francesca Rovera
}

Published online: 4 March 2009

(C) Springer Science+Business Media, LLC 2009

There are very few surgeons, if any, who would still argue that laparoscopic surgery has not been one of the major breakthroughs in the field of surgery during the last three decades. Indeed, in terms of the postoperative course, the "battle" against standard laparotomy and open surgery is largely won. Furthermore, patients' perception and appreciation of reduced traumatic insult is evident, which accounts for the overall increased quality of life after minimally invasive surgical procedures.

During the last few years, attending at various surgical meetings/congresses on both sides of the Atlantic, we have witnessed a virtually unstoppable quest for further improvements to reduce even more the surgical trauma in the field of minimally invasive surgery (MIS). Articles, specific sessions during meetings, surgical societies, and courses on natural orifice transluminal endoscopic surgery (NOTES), single ports, or single access (SPAS) surgery seem to have dominated the scene in recent years [1-6]. Being still relatively young consultant academic surgeons and thus more than interested in new technologies and surgical techniques relevant to minimally invasive surgery and as directors of such MIS and research centers, we have tried our best to embrace the increasing enthusiasm that surrounds NOTES and its variant approaches.

Unfortunately serious concerns about safety and doubts about any real benefits to patient outcome have replaced our initial curiosity for these approaches. During the last EAES Congress in Stockholm, I had the opportunity to attend the specific NOTES session, during which I watched several videos of different procedures performed on human

L. Boni $(\bowtie) \cdot$ G. Dionigi · F. Rovera

Department of Surgical Sciences, Minimally Invasive Surgery

Research Center, University of Insubria, Varese, Italy

e-mail: luigi.boni@uninsubria.it patients: I saw normal-looking appendices and gallbladders removed by a team of three or four different specialists who required some $2-3 \mathrm{~h}$ to perform a procedure, which, even if fully indicated because of symptomatic disease, would normally have been performed by one surgeon and a scrub nurse in less than half an hour. Time, resources, and cost issues immediately came to mind. Even so, further misgivings were raised once I witnessed vaginal, colonic, esophageal, or gastric walls being intentionally perforated and then sutured to carry out relatively simple and straightforward MIS endoscopic operations. Furthermore, all the operations appeared to me and my colleagues to be extremely difficult to perform, largely because of the use of totally inappropriate instrumentation that had been developed for completely different purposes, but which had been modified to meet the new need. In addition we saw cystic ducts clipped and divided without any semblance of anatomical check, or any safety measures to ensure against damage to the common bile duct, or the gallbladder being removed for the hepatic bed in dangerous way, often associated with bleeding or bile spilling. Keep in mind that these were videos selected for an international meeting, and thus more likely to represent the best possible cases performed by these brave and extremely skilled surgeons. At that time we were really surprised that nobody in the audience stood to question the wisdom of all that we were all watching. We left the session with more questions than answers and with an uneasy feeling of not being able to understand what great opportunities we were missing: were we akin to those recalcitrant diehard surgeons who resisted and criticized laparoscopic surgery for so many years after its introduction?

On return to our offices we have since scrutinized the published literature to ascertain the possible future benefits to patient outcome that such difficult and dangerous 
approaches might offer. It appears to us that other than an obvious cosmetic advantage for performing no-scars surgery, NOTES and its variant approaches do not improve the outcome compared with that provided by laparoscopic surgery. Although there is a view (not backed by any robust evidence) that NOTES causes less postoperative pain on the basis that the incision was visceral rather than somatic; even if confirmed (by subsequent studies) would this be sufficient to justify the significant increase in human resource needed, not to mention the substantial increase in costs and increased risks from intentional perforation of hollow organs to obviate the need for the administration of a few analgesic tablets during the postoperative period? Our patients usually go home on postoperative day 1 after laparoscopic cholecystectomy or appendectomy and, if preemptive local anesthetic infiltration of the trocar site is performed, $40 \%$ do not require any postoperative pain killers. Why should we perforate their "natural orifices" and expose them to unnecessary risks to perform a wellstandardized, validated, and cost-effective procedure? Would we have it done to ourselves? The answers to both questions merit a survey amongst MIS surgeons. My answer to both is a definite "no."

To avoid premature judgment on novel procedures, we tried to consider the possible future benefits if and when these surgical approaches will be used for more advanced procedures, such as colorectal resections. In our current practice, the mean hospital stay for patients undergoing laparoscopic colorectal resection is 6 days. This stay has nothing to do with postoperative pain, which is minimal and perfectly controlled by epidural analgesia or i.v. analgesics, but is related to the presence and healing of the anastomosis. In any case we do not see any concrete need to test this procedure on humans because it is evident that at present, there is still a real need for the development of new instruments and technologies for the execution of major operations by these newer approaches. Until such time one has to doubt the ethics of such "human experiments."

More recently one of the authors (LB) attended the American College Clinical Congress in San Francisco, at which quite a large exposure was given to NOTES and even more to single-access or single-port procedures [5]. This variant approach is in part the consequence of the current lack of suitable technologies for the efficient and safe execution NOTES procedures, and in part the reaction by established laparoscopic instrument industries and surgeons to resist the onslaught by NOTES by performing laparoscopic surgery with rigid optics using standard (or modified laparoscopic instruments) through a larger single port introduced through the linea alba via a circum umbilical incision. At this meeting, my first concern was related to the fact that most of the presentations were held by well-known "laparoscopic" surgeons not during formal sessions of the meeting but in the environment of the companies' stands. All the presenting surgeons appeared extremely enthusiastic about their experiences on human beings once they presented their experience with singleport and NOTES procedures. Still there were concerns about safety and real advantages, especially because of the lack of the appropriate technologies. This time the only real advantages that we could ascertain related positively to the medical companies (which were ready to provide the required new disposable products for these procedures) and surgeons' ego. All rules on triangulations, ergonomics, traction and counter-traction, tissue planes, gentle handling of tissues, etc., that we had acquired during our training and that we constantly attempt to teach to our trainees were overlooked by this obsessive fixation with entry into the abdominal cavity through a natural orifices or a single 30$35 \mathrm{~mm}$ hole centered at the umbilicus. What are the benefits? What has happened to Evidence-Based Medicine? Are we in the era of Company Budget Surgery?

Four, robust, large, randomized, clinical trials were needed to convince the surgical community of the benefit (and hence acceptance) of laparoscopic surgery for the treatment of cancer of the large bowel, even when the early videos of these procedures, presented at these same Congresses many years ago clearly demonstrated that the laparoscopic approach respected patient safety and oncologic principles. Rightly, the surgical community needed evidence before it accepted the laparoscopic approach for this common cancer. Now only a handful of surgeons are raising their voices against NOTES and other "ultra" [7] minimally invasive procedures used in humans-and demanding robust evidence for their safety and benefit over laparoscopic surgery. Does this imply acceptance by the establishment or total indifference to these newer MIS approaches?

Thus, we are bewildered and unable to sum up enthusiasm for the new approaches of minimally invasive surgery. Nevertheless, we acknowledge the importance of research and development of novel technologies and new surgical instruments and devices. Only once specific advanced tools become available can we realistically develop new procedures for the treatment of human disorders. We would like to see surgical societies/associations and serious surgical journals in conjunction with established and respected surgical leaders, much more qualified than our group, to comment on meaningful surgical scientific progress and draft guidelines on serious experimental protocols (on inanimate model or animals, not on unsuspecting patients) designed to evaluate new surgical technologies and techniques in independent (with no conflict of interest) academic institutions. In our opinion, the development and validation of new technologies and 
instruments is the key to success for these new fields of surgery.

Arthur L. Bloomfield, outstanding emeritus professor of medicine at the Stanford Medical School in California (USA), once said, "There are some patients whom we cannot help; there are none whom we cannot harm" [8]. We would do well not to overlook this in our quest for surgical progress.

\section{References}

1. Rolanda C, Lima E, Pêgo JM, Henriques-Coelho T, Silva D, Moreira I, Macedo G, Carvalho JL, Correia-Pinto J (2007) Thirdgeneration cholecystectomy by natural orifices: transgastric and transvesical combined approach (with video). Gastrointest Endosc 65(1):111-117
2. Hazey JW, Narula VK, Renton DB, Reavis KM, Paul CM, Hinshaw KE, Muscarella P, Ellison EC, Melvin WS (2008) Natural-orifice transgastric endoscopic peritoneoscopy in humans: initial clinical trial. Surg Endosc 22(1):16-20

3. Gettman MT, Blute ML (2007) Transvesical peritoneoscopy: initial clinical evaluation of the bladder as a portal for natural orifice translumenal endoscopic surgery. Mayo Clin Proc 82(7):843-845

4. Whiteford MH, Swanstrom LL (2007) Emerging technologies including robotics and natural orifice transluminal endoscopic surgery (NOTES) colorectal surgery. J Surg Oncol 96(8):678-683

5. Mutter D, Leroy J, Cahill R, Marescaux J (2008) A simple technical option for single-port cholecystectomy. Surg Innov 15(4):332-333

6. Rao PP, Bhagwat SM, Rane A, Rao PP (2008) The feasibility of single port laparoscopic cholecystectomy: a pilot study of 20 cases. HPB (Oxford) 10(5):336-340

7. Pomp A (2008) Notes on NOTES: the emperor is not wearing any clothes. Surg Endosc 22(2):283-284

8. Cuervo LG, Aronson JK (2004) The road to health care. BMJ 329(7456): $1-2$ 